\title{
Characterization of Boron Containing Graphite Using TEM and EELS
}

\author{
J.S. Kim*, S.J. Kim*, G.H. Kim*, C.H. Chun*, and H.H. Koo* \\ * Materials R\&D Division, Agency for Defense Development, ,Yusung PO Box 35-5, Deajon, 305- \\ 600 , South Korea
}

Boron containing graphites have potential application in high oxidation resistance [1] but carbonbased materials in oxidizing environments show gasification of carbon as carbon oxides [2]. The microstructure of graphite and boron nanoparticles in boron containing pyrographite, with a content of 15 at.\% boron, have been investigated by means of TEM tilting experiment and EELS elemental mapping, respectively. We here report on the new asymmetric structure along rod-axis caused by the orientation-dependent growth of rod-like graphite and on the boron compositional image of boron nanoparticles in every field of view.

A new asymmetric structure in rod-like microsturctures: Two different microstructures are shown in Fig. 1a: the rod-like microstructures are connected each other like a network while the other areas are filled with a matrix-like structure. Their SADPs are shown in Fig. 1b and 1c, respectively, which are typical SADPs with different orientations. In general, the SADPs of pyrographite are similar to those of carbon fiber which has a symmetrical fiber axis normal to c-axis of all graphite sheets, surrounded by closed basal c-planes. In tilting experiments of rod-like structure, an unexpected result was found that the boron containing pyrographite had a $90^{\circ}$ asymmetric structure along the rod axis (Fig. 2 and 3). The rod-like structure tilted at $90^{\circ}$ in Fig. 2 (marked with ' $R$ ') shows high contrast and split c-planes in Fig. 2a but they disappear in Fig. 2b. With increasing tilt angles, the orientation between c-axis of graphite and beam direction is changed from perpendicular and then into parallel. Their consecutive SADPs are shown in Fig. 3. In the tilting experiments of the matrix-like structure, it was revealed as a set of rod-like structures overlapped each other but the contrast disappeared when the c-axis was parallel to the beam direction. The asymmetric rod structures were developed in three dimensional manner so that the bulk sample showed isotropic thermal and mechanical properties.

Observation of boron nanoparticles using EELS: The dark field image cannot avoid excluding graphite diffraction rings even using the smallest objective aperture and its information is restricted within a part of the boron nanocrystalline diffraction spot rings. To remove the diffracted image of graphite and to ensure the boron-compositional image, the boron elemental mapping was conducted using EELS. The boron map is shown in Fig. 4. The boron nanopaticles are uniformly present in both rod- and matrix-like structures, i.e., in all rod-like microstructures when compared with the above tilting experiments. However, the boron influenced on the growth mechanism of the rod-like pyrographite to produce a $90^{\circ}$ asymmetric structure along the rod axis. The boron nanopaticle was identified as $\mathrm{B}_{4} \mathrm{C}$ compound by SADP, XRD, and HREM image.

References

[1] L.E. Jones and P.A. Thrower, J. de Chim. Physique 84, (1987) 1431.

[2] J.E. Sheehan, Carbon 27 (1989) 709. 

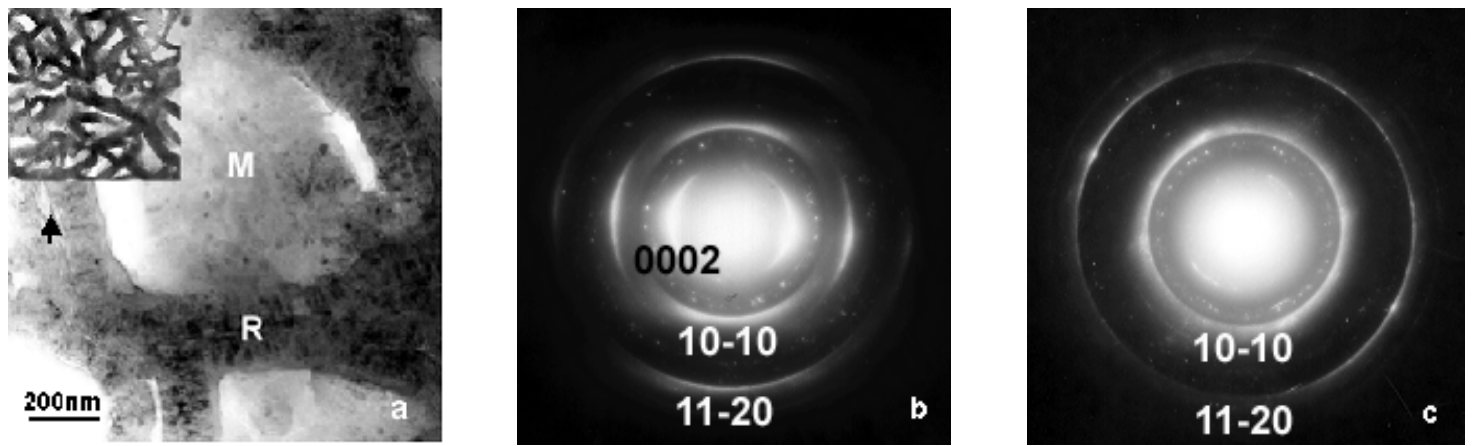

FIG. 1. (a) TEM image and inserted macro image of boron containing pyrographite, and its SADPs of (b) rod-like and (c) matrix-like microstructures. R: rod-like, M: matrix-like microstructures. The arrow in (a) shows split c-planes which are parallel to the rod growing axis.
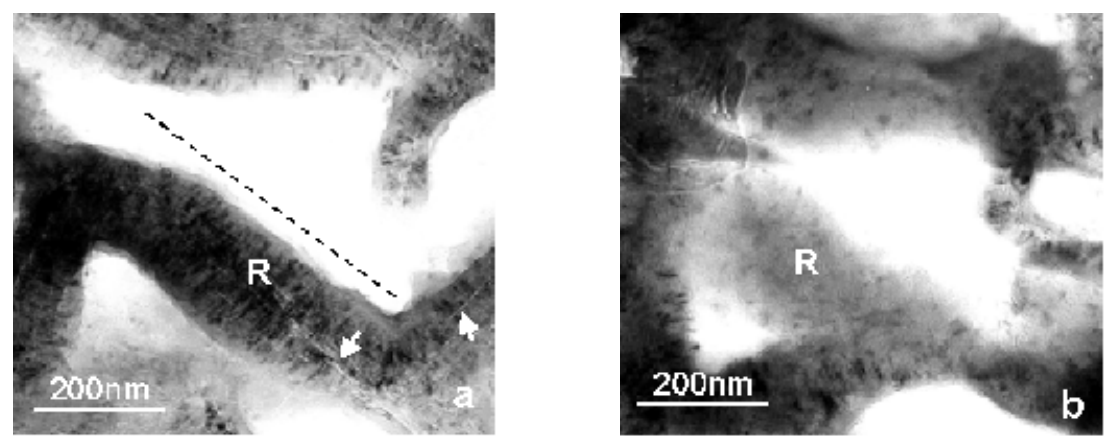

FIG. 2. Rod-like microstructure (marked with 'R') was tilted at $90^{\circ}$ along rod axis (dash line) : (a) $45^{\circ}$ tilt, (b) $+45^{\circ}$ tilt. Arrows: split c-planes.
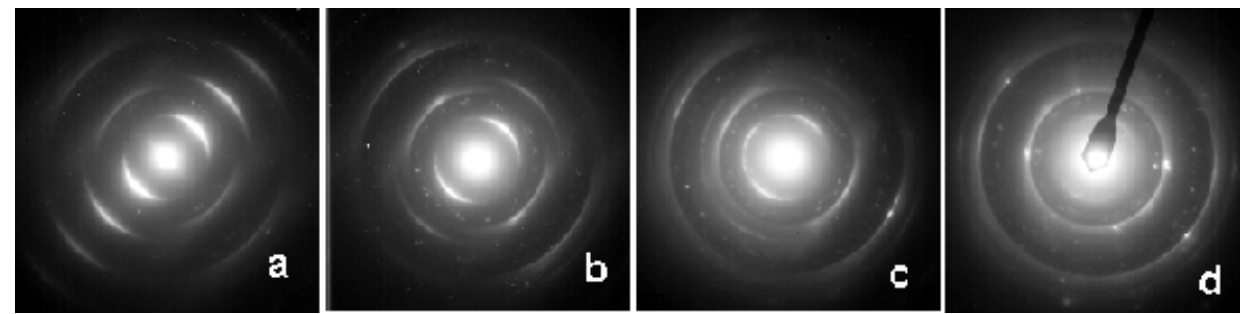

FIG. 3. The SADPs from the mark 'R' in Fig. 2 when the rod-like structure was tilted along the rod axis (dash line): (a) $-45^{\circ}$ tilt, (b) $-20^{\circ}$ tilt, (c) $+20^{\circ}$ tilt, and (d) $+45^{\circ}$ tilt.
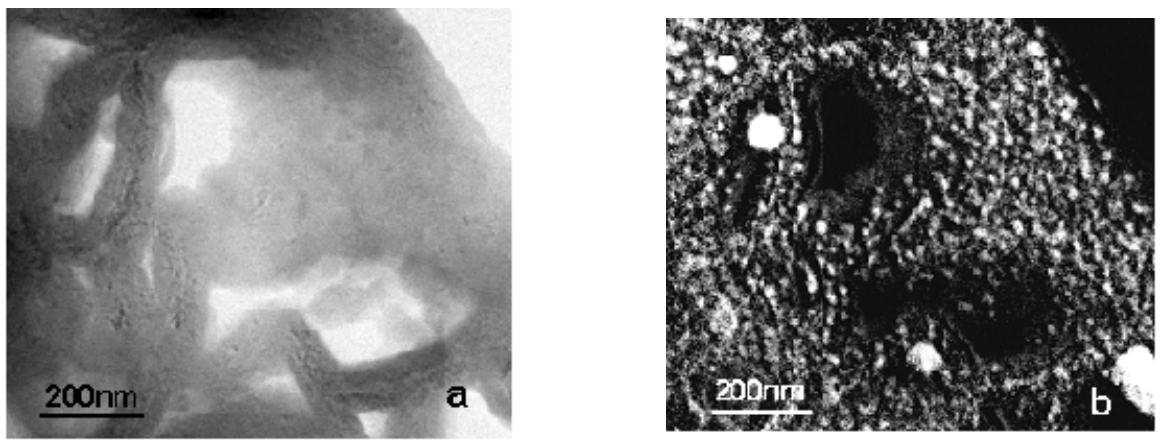

FIG. 4. TEM image (a) and its boron elemental map using EELS (b). Note that the boron nanopaticles are present in both rod- and matrix-like structures as a size of about $40 \mathrm{~nm}$ in diameter. 